\title{
Membrane Part
}

National Cancer Institute

\section{Source}

National Cancer Institute. Membrane Part. NCI Thesaurus. Code C38627.

Any component of the thin sheet or layer of pliable tissue, serving as a covering or

envelope of a part, as the lining of a cavity, as a partition or septum, or to connect two structures. 